\title{
Electromechanical simulation of a Pointing System Model for Satellite Optical communication
}

This paper was downloaded from TechRxiv (https://www.techrxiv.org).

LICENSE

CC BY 4.0

SUBMISSION DATE / POSTED DATE

26-02-2022 / 03-03-2022

\section{CITATION}

Del Mondo, Federico; Gregorio, Anna; Seriani, Stefano; Gallina, Paolo (2022): Electromechanical simulation of a Pointing System Model for Satellite Optical communication. TechRxiv. Preprint.

https://doi.org/10.36227/techrxiv.19242783.v1

$\mathrm{DOI}$

10.36227/techrxiv.19242783.v1 


\title{
Electromechanical simulation of a Pointing System Model for Satellite Optical Communication
}

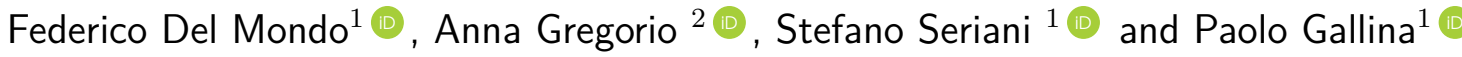 \\ ${ }^{1}$ Department of Engineering and Architecture, University of Trieste, Via A. Valerio 6/1, 34100 Trieste, Italy; \\ ${ }^{2}$ Department of Physics, University of Trieste, Via A. Valerio 2, 34100 Trieste, Italy; \\ federicodelmondo@studenti.units.it (F.DM.); anna.gregorio@ts.infn.it (A.G.); sseriani@units.it (S.S.); pgallina@units.it (P.G.)
}

February 26, 2022

\begin{abstract}
The increasing of satellite's infrastructure and laser telecom systems on board small satellites prompted us to investigate the feasibility of a ship's autonomous navigation by a two Degree of Freedom (DoF) Pointing System (PS) located on ship's deck. We analyzed in detail the dynamics of the system dealing with the design and control system of the model. Ship motions and satellite's coordinates with relative errors (acquisition errors for ship motions, acquisition and orbital propagation errors for satellites) were considered during simulations. Initial results led us to adopt scanning techniques in order to increase the achievement of satellite's locking. By evaluating the initial results and those referred to the scanning, the satellite's locking is feasible in case such scanning techniques are adopted. The trend of results is similar for all given ship motions scenarios and scanning techniques adopted, since the acquisition's error for ship motions together with errors of acquisition and orbital propagation for the satellite affect the results, unlike the ship motions. A secondary fine-motion actuating system for scanning strategies is described.
\end{abstract}

Keywords: Pointing System - ATP - Ship Motions

\section{Introduction}

The concept of autonomous navigation represents an innovative idea that will surely find a practical field of application in the near times and that will completely change the traditional concept of current maritime navigation. The ability of a ship to control its own actions independently while transporting goods from one port to another is referred to as autonomous shipping [1]. MUNIN described autonomous ships as the 'next generation modular control systems and communications technology that will enable wireless monitoring and control functions both on and off the board. These will include advanced decision support systems to provide a capability to operate ships remotely under semi or fully autonomous control' [2].

Quantum communication in reference to satellites and Cubesat (small satellites) is not new [3] but this work address the problem of a direct link between two crucial topics in the context of the European efforts: autonomous navigation and quantum communication in space, thus securing a critical infrastructure by using small satellites. Quantum Key Distribution (QKD) is a relatively new cryptographic primitive for establishing a private encryption key between two parties. The first proposal regarding this technology was born in 1984 [4]. The Free-space Quantum Key Distribution (QKD) was first implemented over an optical path of about $30[\mathrm{~cm}]$ in 1991 and in subsequent years many achievements have been made in satellite to ground [6][7] and ship to ship communication [8] [9]. However, currently, most of the experiments only involve space and ground communication links or communication between ships. Since water bodies occupy more than $70 \%$ of the Earth's surface, the global-scale communication network tends to extend these technology in the feature from the ground to the sea. High volumes of data need to be transmitted by satellite and for this there is an urgent need to conduct simulations and experimental verifications of laser communication between satellites and ships at sea. Acquisition, tracking, and pointing (ATP) is the key technology used in laser communication, which requires the rapid acquisition and alignment of line-of-sight between two platforms and the establishment of communication links with high precision, high probability, and high dynamic tracking. High pointing accuracy is difficult to achieve for shipborne ATP because of ship motions, which poses a higher challenge to this technology [10].

In this work, we consider the ATP systems as the dynamic core of the pointing System (PS) model, capable of simulating and foreseeing the dynamic behavior of the interconnected modules. The pointing System model has 2 Degree of Freedom (DoF) [Fig:1]. 


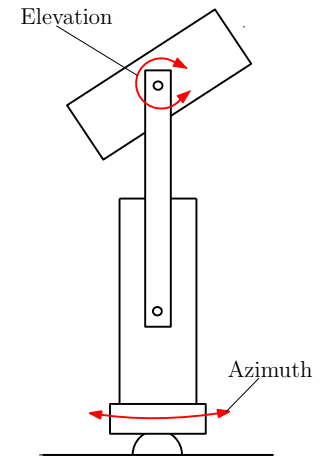

Figure 1. Model DoF.

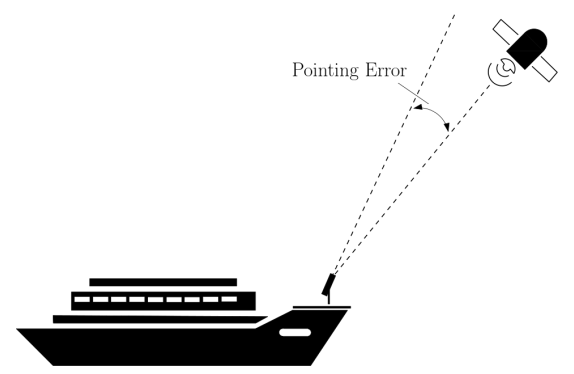

Figure 2. Pointing Error.

The main part of the research refers to the control system generation together with the dynamic simulation and scanning strategies to reduce the pointing error [Fig:2], on a range of 100 [ $\mu \mathrm{rad}$ ] for satellite's locking. Low orbit satellites and different scenarios of ship motions and their influence on a shipping vessel (Euler displacement) have been considered to introduce the basal oscillation of the pointing system model. The acquisitions error for ship motions together with errors of acquisition and orbital propagation for the satellite have been inserted into the control block diagram and their affect considered. Due to the high frequencies required to increase the pointing accuracy with scanning strategies, a scanning module provided with piezoactuators integrated with the optical system is introduced.

\section{Methodology}

The PS is placed on vessel's deck and should be able to point satellite's to establish communication links.

\subsection{Pointing System Model}

The model characteristics of the PS are:

\begin{tabular}{ll}
\hline Characteristics & Description \\
\hline Model DOF & Azimuth \& Elevation \\
Mass & $\simeq 5[\mathrm{~kg}]$
\end{tabular}

Table 1: Model characteristics.

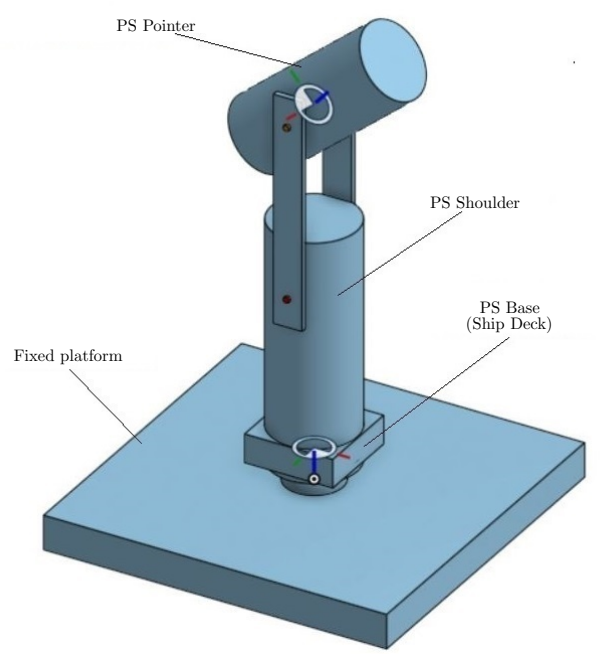

Figure 3: Pointing System model. 


\subsection{Reference Systems}

The satellite's coordinates are expressed in Earth-Centered Earth-Fixed (ECEF) reference frame; geocentric coordinates can be used for locating astronomical objects in the Solar System in three dimensions along the Cartesian X, Y, and Z axes. The ECEF is a coordinate frame that doesn't suffer some of the geometric issues, especially in polar regions, of a geographical coordinate frame that might define position in terms of latitude, longitude, and some height measure [11] . Earth reference system is a Cartesian coordinate system based on the tangent plane on the Earth's surface and the perpendicular local vertical direction. The origin of Earth reference frame is coincident to the ship location and given by the GPS coordinates.

Ship reference system is a Cartesian coordinate system linked to the boat and located on the ship deck, required to insert the PS base oscillations due to sea conditions and ship speed.

Optical reference frame has it's origin in the baricentre of the optical component with the $\mathrm{X}$ axes direction along the line-of-sight.

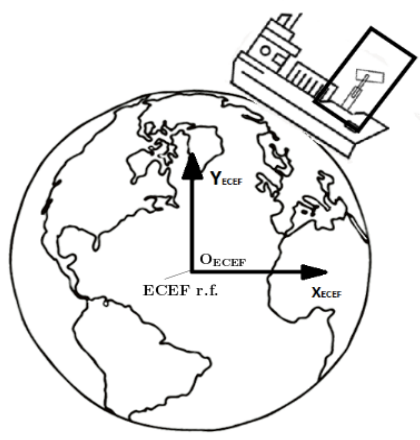

(a) ECEF reference system

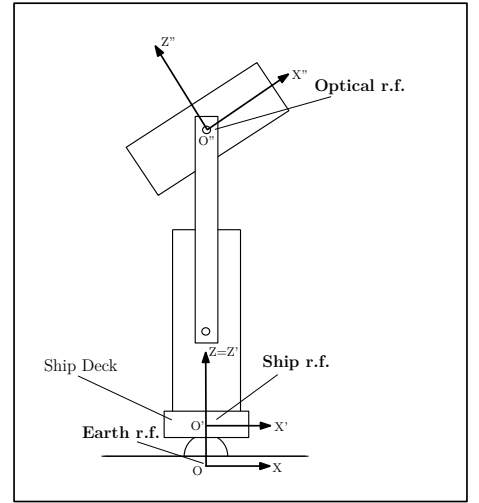

(b) PS reference system.

Figure 4: Reference systems overview.

\subsection{Satellite's coordinates and Ship position}

As introduced above, the satellite's coordinates are expressed in the ECEF reference frame:

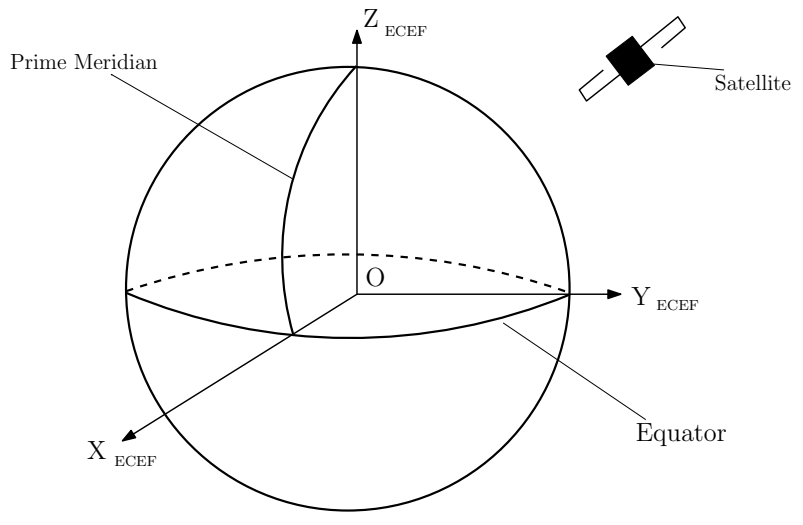

Figure 5: Satellite's coordinates.

First we can define the features of low orbits satellite's taken into consideration:

\begin{tabular}{ll}
\hline Parameter & Mean Value \\
\hline Earth distance & $631.7[\mathrm{~km}]$ \\
Speed & $4.2[\mathrm{~km} / \mathrm{s}]$
\end{tabular}

Table 2: Satellite's parameters.

with an orbital period of 180 [min]. 
Therefore, it is possible to estimate a random distribution of the error in X-Y-Z directions [13] [14] with a time step of $0.5[\mathrm{~s}]$ in the following intervals:

\begin{tabular}{ll}
\hline Direction & Range $[\mathrm{km}]$ \\
\hline $\mathrm{X}$ & $-0.354: 0.353$ \\
$\mathrm{Y}$ & $-0.729: 0.728$ \\
$\mathrm{Z}$ & $-0.661: 0.661$
\end{tabular}

Table 3: Randomic error definition.

Should be necessary transform [12] these satellite's coordinates in the Earth reference system using the GPS vessel's coordinates for the subsequent control system generation. The Earth reference system will be considered an absolute reference frame for ship motions and optical component displacement definition.

The ship's position is given in Geodetic coordinates, these define the origin of the Earth reference system or otherwise, can be useful for the transformation of the satellite's coordinates from ECEF to Earth reference systems.

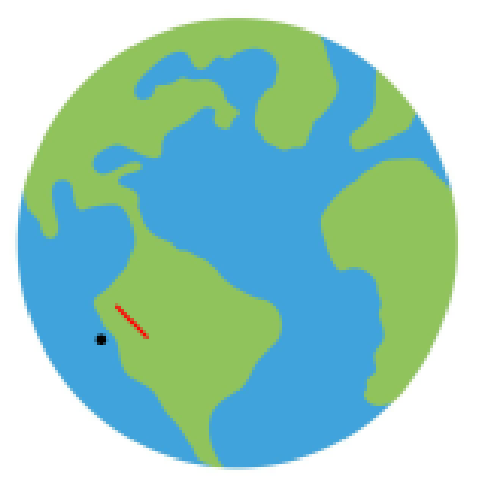

Figure 6: Satellite's trajectory (red) and ship position (black).

\subsubsection{Satellite's coordinates transformation from ECEF to Earth reference frame}

It is possible to define the point $\mathrm{P}$ as the location of the ship on Earth, given by ship's GPS data in geodetic coordinates ( $\varphi$ latitude, $\lambda$ longitude, $h$ altitude ). The same point $P$ is coincident with the origin of the Earth reference frame $\left(\mathrm{X}_{\text {Earth }}, \mathrm{Y}_{\text {Earth }}, \mathrm{Z}_{\text {Earth }}\right)$ while, the satellite's coordinates are expressed in the ECEF reference frame $\left(\mathrm{X}_{\mathrm{ECEF}}, \mathrm{Y}_{\mathrm{ECEF}}, \mathrm{Z}_{\mathrm{ECEF}}\right)$.

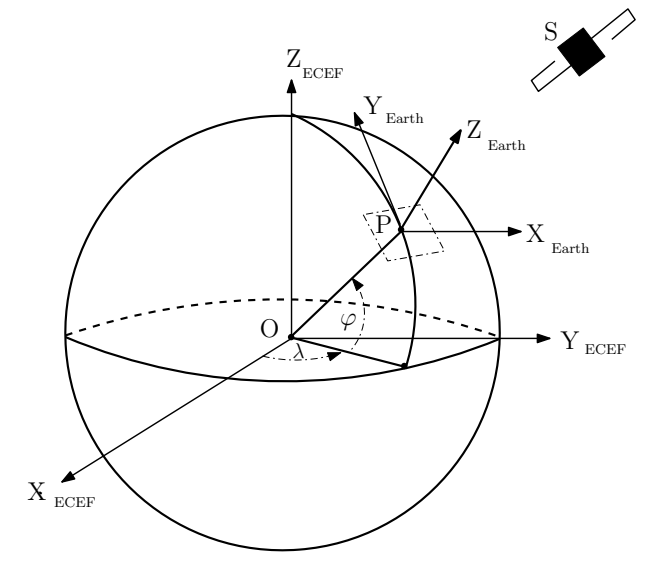

Figure 7: Coordinates transformation scheme.

Satellite Coordinates - ECEF r.f. :

$$
S_{E C E F}=\left(S_{X}, S_{Y}, S_{Z}\right)
$$

Ship Coordinates - Geodetic r.f. :

$$
P_{G E O}=(\varphi, \lambda, h)
$$


To define the displacement of the ship position and the satellite (displacement of Earth r.f relative to Satellite r.f), the geodetic coordinates of ship location should be transformed in the ECEF reference frame [15].

Ship Coordinates - ECEF r.f. :

$$
P_{E C E F}=\left(P_{X}, P_{Y}, P_{Z}\right)
$$

With the following matrix transformation:

$$
\left[\begin{array}{l}
X \\
Y \\
Z
\end{array}\right]=\left[\begin{array}{ccc}
-\sin (\lambda) & \cos (\lambda) & 0 \\
-\sin (\varphi) \cos (\lambda) & -\sin (\varphi) \sin (\lambda) & \cos (\varphi) \\
\cos (\varphi) \cos (\lambda) & \cos (\varphi) \sin (\lambda) & \sin (\varphi)
\end{array}\right]\left[\begin{array}{l}
S x-P x \\
S y-P y \\
S z-P z
\end{array}\right]
$$

it is possible to define the satellite's coordinates from the Earth reference frame:

$$
S_{\text {Earth }}=(X, Y, Z)
$$

\subsection{Ship Motions}

The Pointing System (PS) is fixed on the ship [16] [17] [18] and as the ship moves due to sea waves, the PS is affected by yaw, pitch, and roll disturbances, which cause the PS's line-of-sight axis to oscillate.

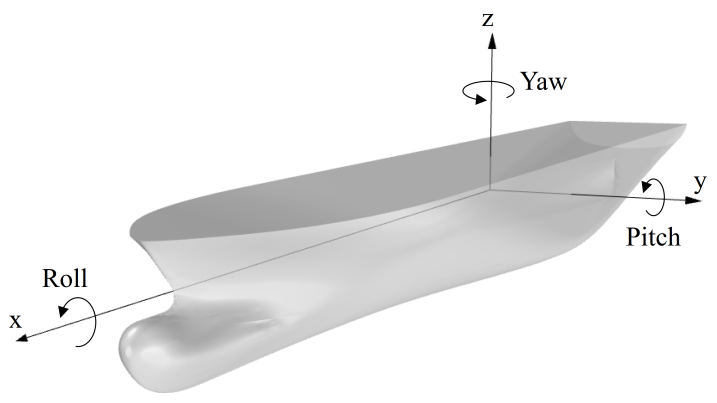

Figure 8: RPY convention - Ship r.f. .

For the simulation, four severe scenarios have been defined, based on the ship characteristics and on the environmental conditions from IACS Rec.34 [19] [20], as reported in Table 4. Long crested waves have been used, assuming a Bretschneider spectrum. Ship motions have been predicted by a linear seakeeping approach that was also used in past studies (e.g. $[21][22])$.

\begin{tabular}{llllll}
\hline Scenario & Heading $^{1}$ & Roll Period $^{2}[\mathrm{~s}]$ & Ship speed $[\mathrm{kn}]$ & Peak period $^{3}[\mathrm{~s}]$ & SWH $^{4}[\mathrm{~m}]$ \\
\hline 01 & $90^{\circ}$ Beam Sea & 19.6 & 0 & 23.2 & 14.3 \\
02 & $90^{\circ}$ Beam Sea & 19.6 & 0 & 16.2 & 10.8 \\
03 & $180^{\circ}$ Head Sea & 12.6 & 5 & 20.4 & 13.1 \\
04 & $180^{\circ}$ Head Sea & 12.6 & 10 & 20.4 & 13.1
\end{tabular}

Table 4: Overview of scenarios for ship motions prediction.

1 Heading is intended here as the relative angle between the direction the vehicle/vessel is pointing to, and the propagation direction of waves.

2 The time necessary for one complete double oscillation or roll of a ship as from port to starboard and back to port.

3 Period corresponding to the peak of the sea spectrum.

4 In physical oceanography, the significant wave height (SWH) is defined as four times the standard deviation of the surface elevation. 
Ship motions will be taken into account considering only angular motions, i.e. Roll, Pitch and Yaw. Examples of calculated ship motion time histories are shown in Figure 9. The plots represent the estimated ship motions (measured by simulated angular sensors present on the ship) versus time.

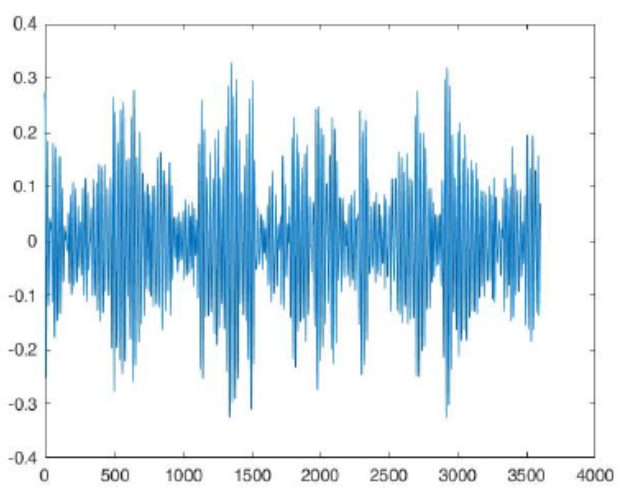

(a) Roll.

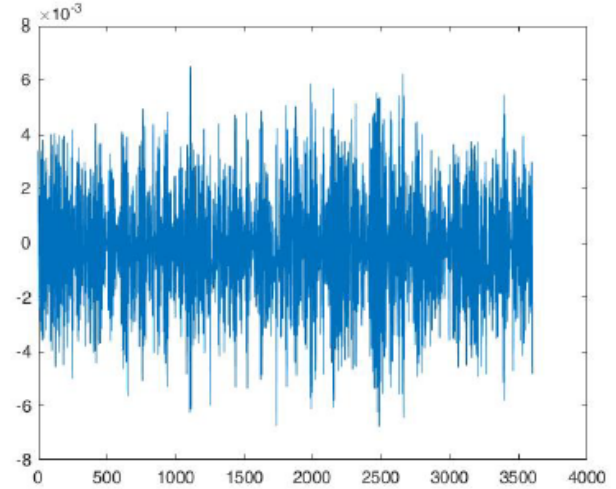

(b) Pitch.

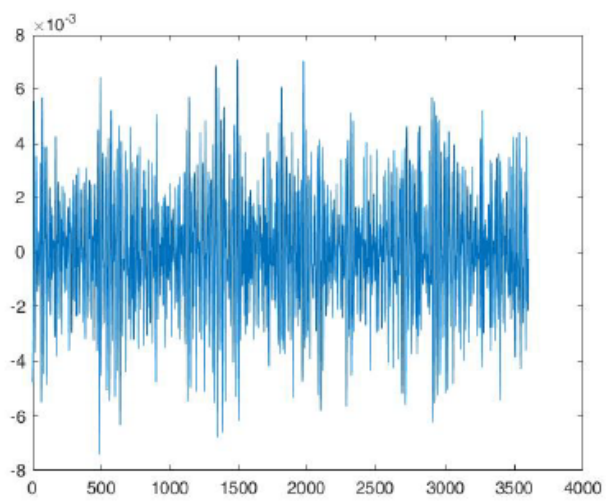

(c) Yaw .

Figure 9: Ship motions overview .

The ship motions errors can be expressed in terms of RMS quantities [10]:

\begin{tabular}{ll}
\hline Angle & RMS Value $[\mathrm{mrad}]$ \\
\hline Roll & 0.09 \\
Pitch & 0.09 \\
Yaw & 0.18
\end{tabular}

Table 5: Ship motions acquisition errors.

These can be expressed in terms of azimuth and elevation [Fig:1] errors for the PS, resulting a random distribution with zero mean and defined variance [10]:

\begin{tabular}{lll}
\hline DOF & Variance $\left[\mathrm{mrad}^{2}\right]$ & Mean $[\mathrm{mrad}]$ \\
\hline Azimuth & 0.18 & 0 \\
Elevation & 0.09 & 0
\end{tabular}

Figure 10: Ship motions errors in terms of Azimuth and Elevation. 


\subsection{Control Scheme Overview}

It is possible to introduce a control scheme overview for the dynamic simulation:

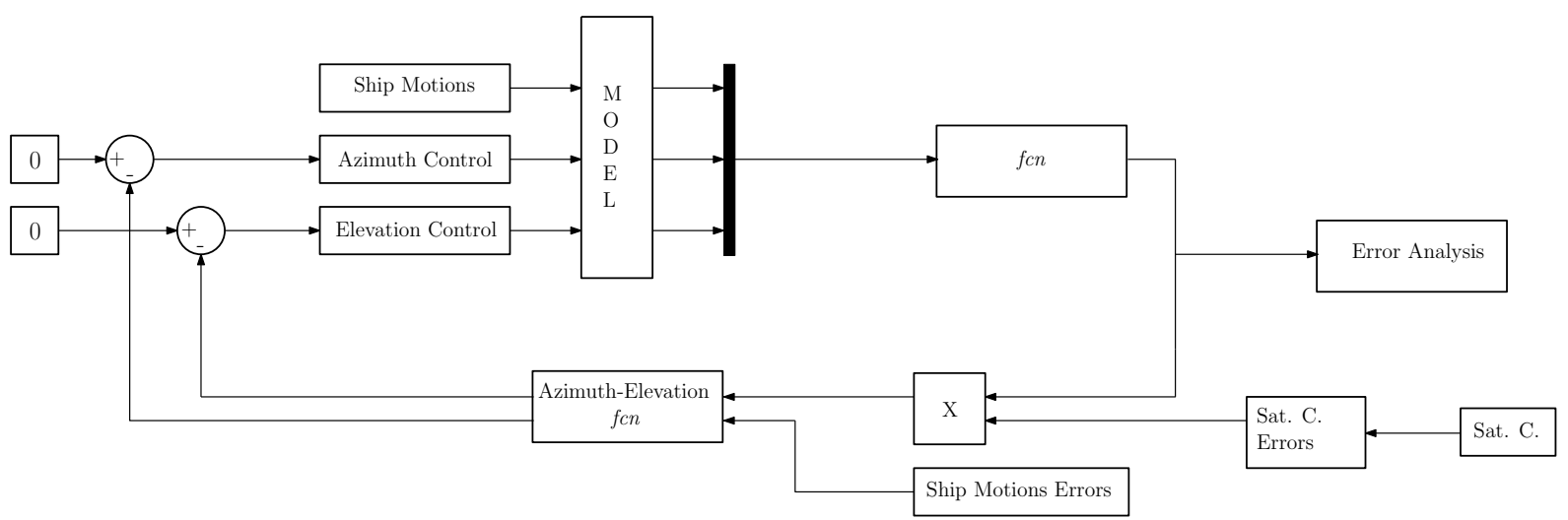

Figure 11: Control diagram overview.

The voluntee is to perform an ADAMS-Simulink co-simulation with the model exported from ADAMS into a Simulink block as shown in Fig:11; the model features will be explained in the next chapter.

Ship motions introduce an angular displacement of the PS base (Fig:3) and together with the Satellite coordinates from Earth reference system are both input of the control system.

The Azimuth-Elevation function provides to the generation of quantities proportional to the amount of satellite displacement for PS motion.

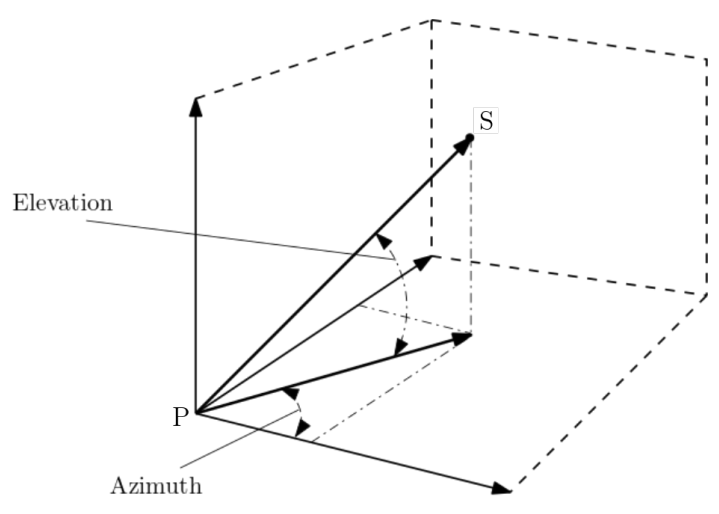

Figure 12: Azimuth and Elevation.

$$
\begin{gathered}
A z=\operatorname{atan} 2(y, x) \\
E l=\operatorname{atan} 2\left(z, \sqrt{x^{2}+y^{2}}\right)
\end{gathered}
$$

The signal obtained is compared with a null reference signal so that guarantees a continuous update with respect to the previous value of the PS torque input signal. The right side of the control block diagram concerns the pointer motion to evaluate the feasibility of satellite's pointing with the adoptable technology of the optical component.

In the control diagram there are also considered the errors of acquisition of ship motions and the errors of acquisition and orbital propagation of the satellite. 


\section{Dynamic Simulation Setup}

In order to analyze the feasibility of pointing passing through the pointing error analysis, a simulation using the commercial software MSC ADAMS is performed.

The model composed by ideal bodies (Fig:3) is imported (e.g. in Parasolid) in MSC ADAMS and inertial properties are applied coherently with the possible experimental setup. Appropriate kinematic joints connect the separate components; the final model and relative input-output variables can be seen in Figure 13a.

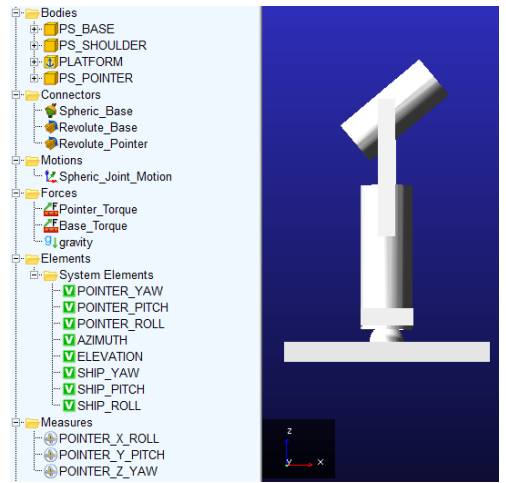

(a) PS ADAMS model.

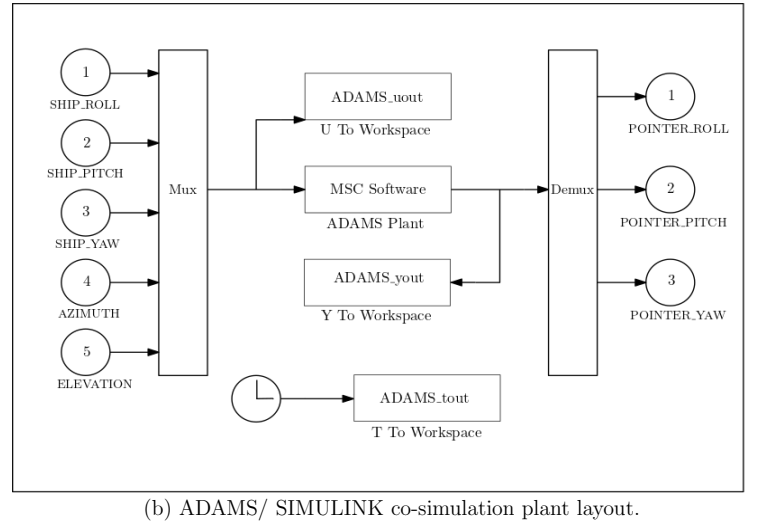

(b) ADAMS/ SIMULINK co-simulation plant layout.

Figure 13: ADAMS/SIMULINK co-simulation setup.

Hence, the satellite's coordinates, ship motions and relative errors are introduced around the model box like shown in the previous chapter (Fig:11).

The simulations are conducted in the context of the ADAMS/SIMULINK co-simulation. The modeled torques and ship motions Euler's angles are the controllable variable that are exported as an input state variables for the ADAMS/SIMULINK co-simulation. The model output is the angular displacement of the Pointing reference system compared to a fixed reference frame (Earth r.f.).

In order to enable the multi-body simulation, the ADAMS plant is exported, and simulations are conducted through Simulink. In particular, the Simulink model setup can be seen in Figure 13b.

Simulation setting:

\begin{tabular}{ll}
\hline Variable & Value \\
\hline Sim. Time & $10[\mathrm{~s}]$ \\
Sim. Time Step & $0.001[\mathrm{~s}]$
\end{tabular}

Table 6: Simulation setup.

For the simulation, relative position between ship and satellite is the one shown in Figure 6.

\subsection{Simulation features}

- The azimuth and the elevation axes are concurrent in a single point. Therefore, as concerns the ship motions, it will be enough to consider only the angular displacement, while the translational one will be negligible;

- Pointing System mass $\simeq 5[\mathrm{~kg}]$;

- Optical components that will be introduced in next chapters have negligible mass compared to the PS mass.

\section{Pointing Error}

The main part of the research is the pointing error evaluation and if the system would be able to accurately point the satellite, we would expect zero error in pointing assessment.

The pointing accuracy is evaluated directly in the control block diagram (Fig:11) and a post processing data analytics will be necessary for results evaluation.

What is expected is a no-zero pointing error for the ship motions presence, that introduce the PS swinging, and also for the acquisitions error for ship motions together with errors of acquisition and orbital propagation for the satellite.

Therefore, a maximum value in terms of pointing error must be introduced to verify the establishment possibility of a communication link between ship and satellite.

The laser beam used in space laser communication is emitted at an angle that is near the diffraction limit. 
The divergence angle of very narrow communication beams requires both communicating sides to maintain extremely high dynamic alignment accuracy.

Since a constant and perfect alignment will be difficult to achieve, the main goal is to to lock the satellite and then follow its orbital propagation. In order to lock the satellite and enable a quantum communication is required a pointing error less than $100[\mu \mathrm{rad}]$.

The Pointing error can be expressed in terms of Azimuth and Elevation:

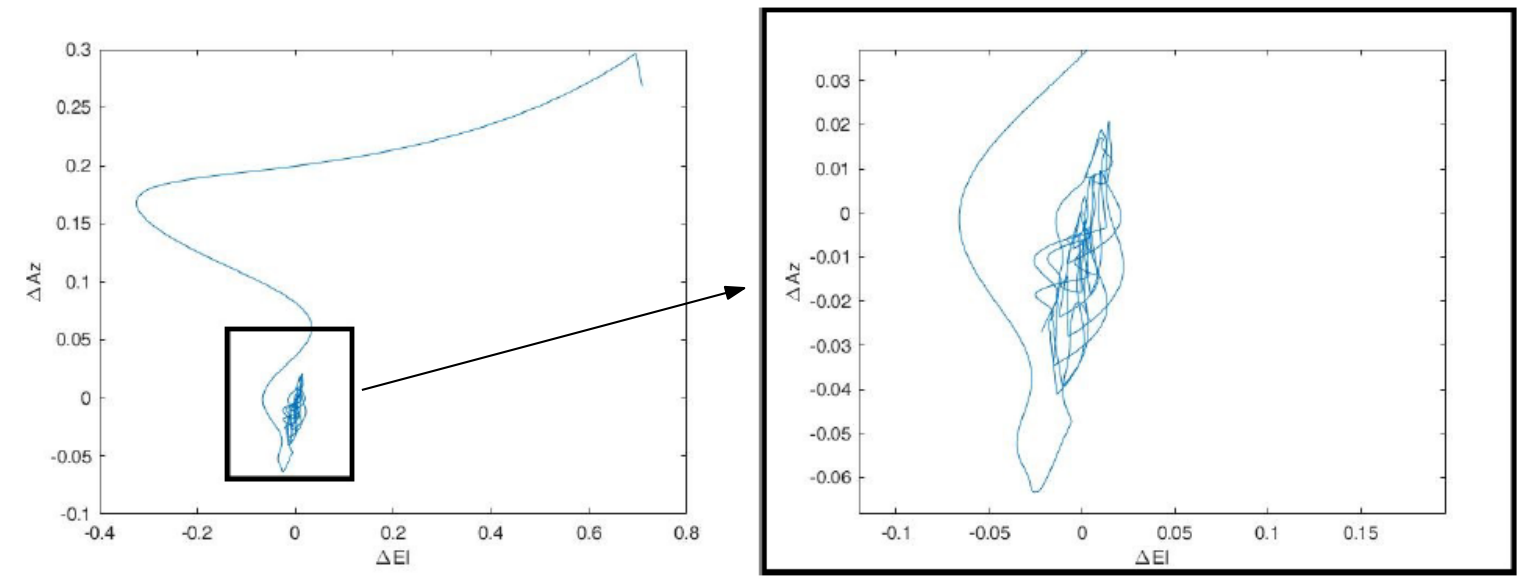

Figure 14: Pointer motion

The upper side of the pointer motion plot (Fig:14 left side) corresponds to the initial displacement of the system from the initial configuration to the effective pointing condition.

If the satellite was pointed with zero error the curve should tend to zero but, this does not happen due to the presence of ship motion and errors introduced in the previous chapters.

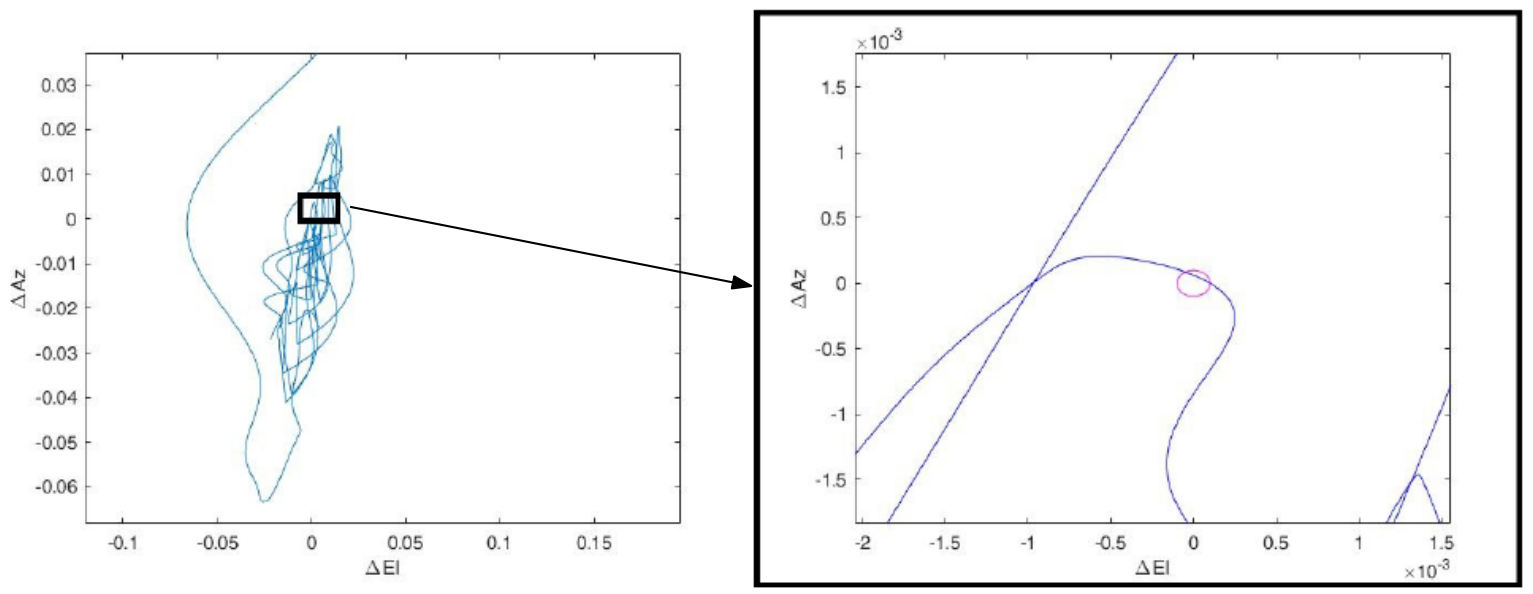

Figure 15: Pointer motion and locking area (red).

To enable the satellite pointing, the error must be less than $100[\mu \mathrm{rad}]$, so we can introduce the locking area (red). In Figure 15 the possibility to lock the satellite happens only one time in 10 seconds but, this is function of the ship motion scenario considered for the evaluation.

For this reason, it is possible that with another ship motion scenario this not happen and the system may not reach the satellite lock.

Therefore can be helpful to adopt stretegies to increase the satellite's locking; this strategies can be named as scanning strategies and consists in the pointer rotation around the desired position to point with the aim to limit acquisition errors of ship motions and satellite's orbit.

\subsection{Scanning strategies}

The scanning strategy aims at reducing the pointing error and ensures that the locking takes place in all conditions.

Different scanning techniques can be considered and then analyze if these improve the pointing accuracy. In this study, 
we have considered the Conical scanning and the Bernulli's Lemniscate scanning. Since we expect to use high scanning frequencies, the scanning methodologies will be implemented using the optical system's internal mirror linked to four actuators.

\subsubsection{Conical Scanning}

Conical scanning is performed by adding two signals to the original signal of pointer motion: one sine wave and the other cosine wave with a specific amplitude $\left(r_{s}\right)$ and frequency $(\omega)$. Actually this movement can be carried out by the mirror inside the optical system, so, there will be constraints in the choice of the values of $r_{s}$ and $\omega$.

Too high values of $r_{s}$ will lead to a movement not feasible by the system and too high values of $\omega$ may not be necessary for the locking to take place. Similarly, too low values of $\omega$ will not guarantee a Conical scanning and too low values of $r_{\mathrm{s}}$ will not guarantee the satellite's locking.

Considering the pointer motion coordinates:

$$
P=\left(P_{x}, P_{y}\right)
$$

it is possible to define Conical scanning coordinates:

$$
\begin{aligned}
& H_{x}=P_{x}+r_{s} * \cos (w t) \\
& H_{y}=P_{y}+r_{s} * \sin (w t)
\end{aligned}
$$

and then we can plot the new pointer motion (fine motion in case of scanning) (black) over the previous one (blue):

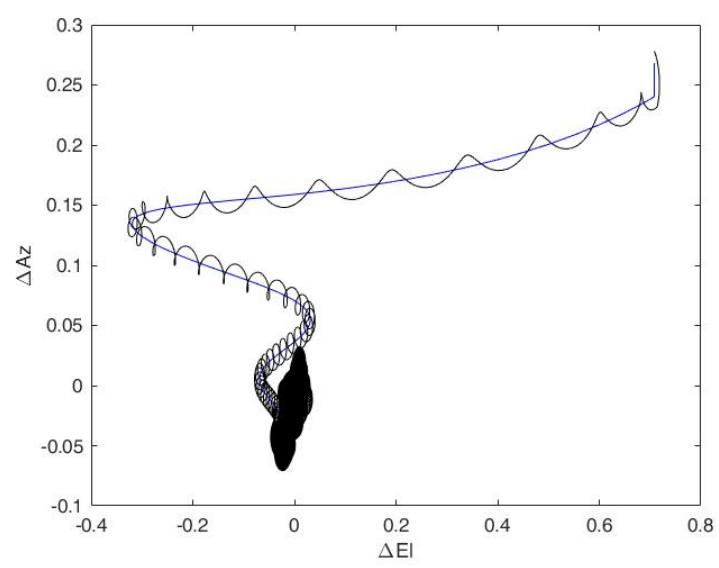

Figure 16: Conical scanning motion.

Evaluating now the locking range to enable the locking:

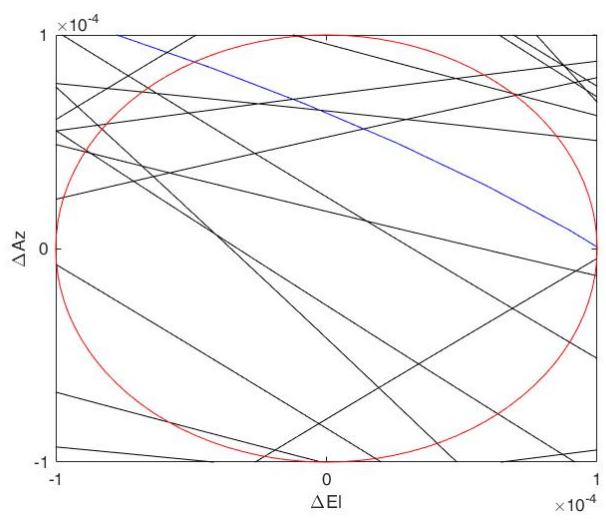

Figure 17: Transitions over the lock range.

We can consider the number of transitions such as the number of times in which the pointing error is less than the maximum targeting error.

The number of transitions within the area of minimum error increases significantly as the parameters $r_{\mathrm{s}}$ and $\omega$ changes. In particular, the number of transitions increases considering low $r_{s}$ values and high $\omega$ (frequency) values. 


\subsubsection{Bernoulli's Lemniscate Scanning}

Another scanning method consists in the use of the Bernoulli Lemniscate.

The aim is to verify that there is another feasible scanning technique able to engage the satellite with a greater probability, that is, an increase in the number of transitions in the lock range.

In geometry, the Lemniscate of Bernoulli is a plane curve defined from two given points F1 and F2, known as foci, at distance $2 c$ from each other as the locus of points $P$, so that $P F_{1} \cdot P F_{2}=c^{2}$.

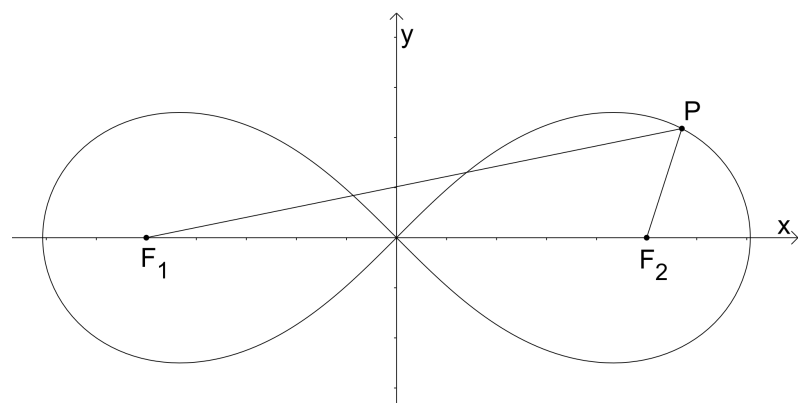

Figure 18: Bernoulli's Lemniscate.

The equations can be stated in terms of the focal distance $c$ or the half-width a of a lemniscate. These parameters are related as $\mathrm{a}=\mathrm{c} \cdot \sqrt{2}$.

Considering the pointer motion coordinates:

$$
P=\left(P_{x}, P_{y}\right)
$$

it's possible to define the Lemniscate scanning coordinates:

$$
\begin{gathered}
L_{x}=P_{x}+\frac{a * \cos (w t)}{1+\sin ^{2}(w t)} \\
L_{y}=P_{y}+\frac{a * \sin (w t) * \cos (w t)}{1+\sin ^{2}(w t)}
\end{gathered}
$$

Then, the new pointer motion (black) can be plotted over the previous one (blue):

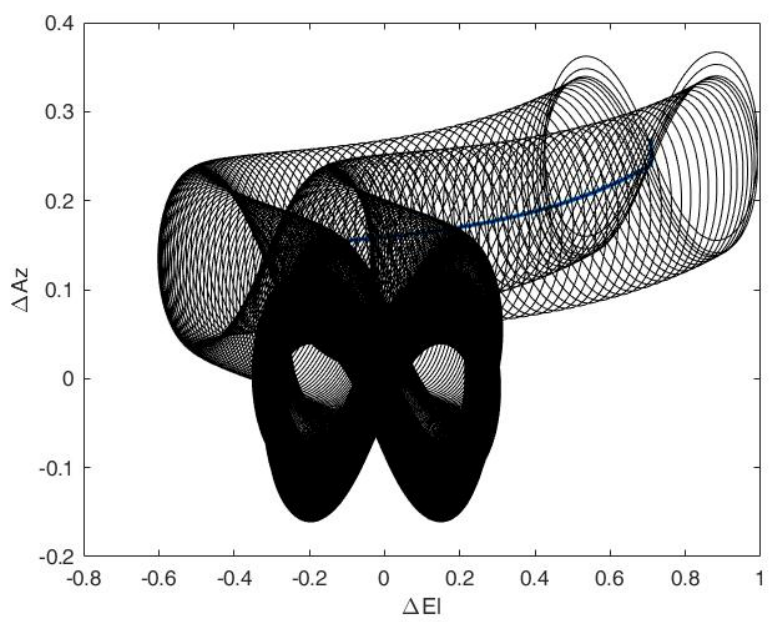

Figure 19: Lemniscate scanning motion.

\section{Results}

The aim now is to evaluate the results with two different scanning techniques and the four ship motions scenarios introduced in the previous chapter.

The mirror inside the optical system that has to carry out the locking with the Conical or Bernoulli Lemniscate techniques, will be set in motion by four piezoelectric actuators.

The parameters $r_{s}$ (or $a$ ) and $\omega$ will be used for the choice of the piezoelectric actuators. 


\subsection{Conical scanning}

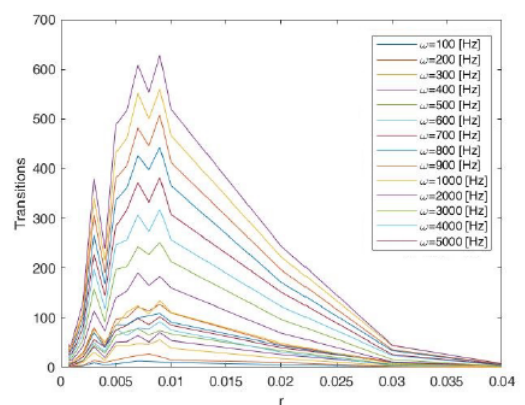

(a) Scenario 01 .

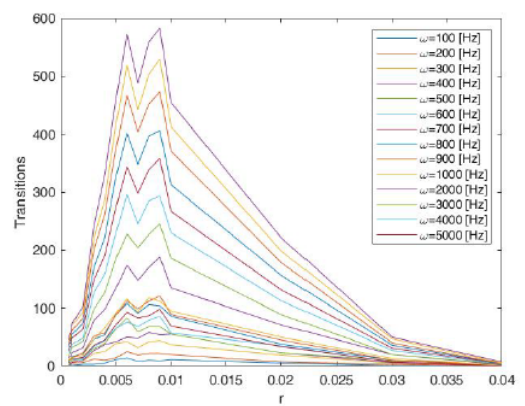

(c) Scenario 03.

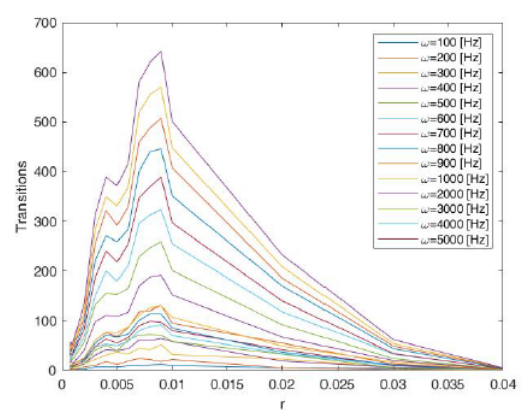

(b) Scenario 02 .

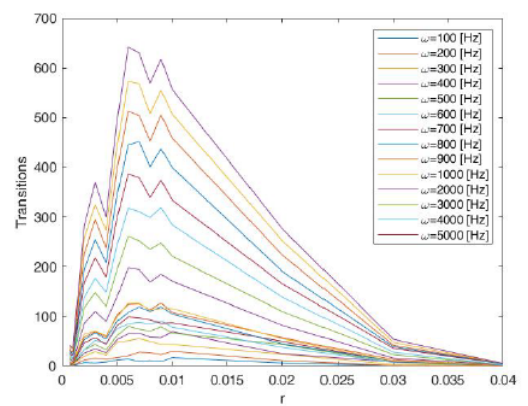

(d) Scenario 04 .

Figure 20: Scenario results overview.

As $\omega$ increases, the number of transitions increases and all the scenarios show a maximum number of transitions for approximately $r_{s}=0.009$ [rad].

\subsection{Bernoulli's Lemniscate scanning}

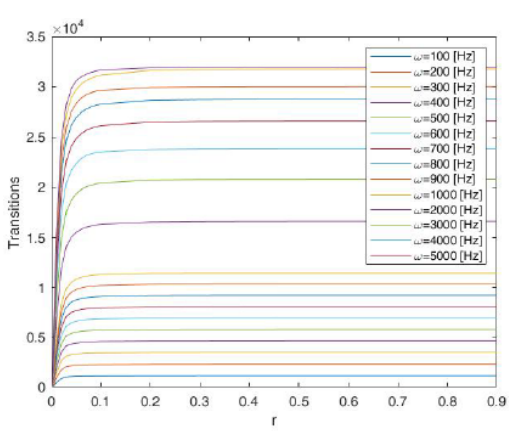

(a) Scenario 01 .

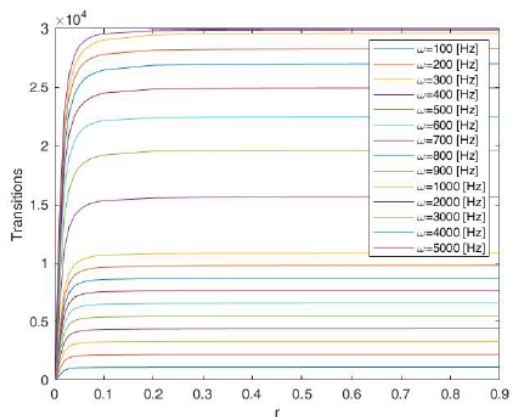

(c) Scenario 03 .

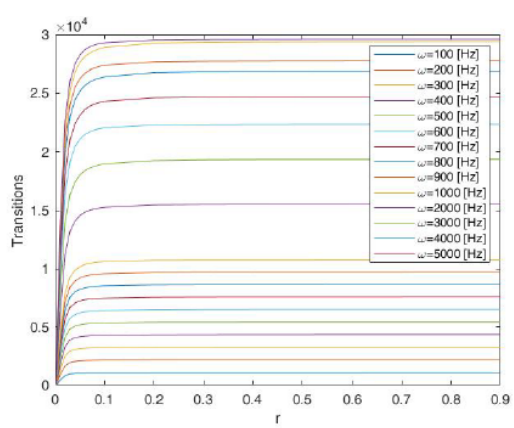

(b) Scenario 02 .

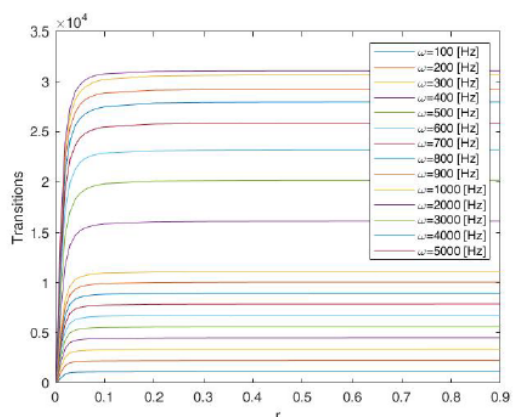

(d) Scenario 04 .

Figure 21: Scenario results overview. 
As in the case of Conical scanning, the number of transitions increases as the frequency increases.

The large increase in the number of transitions in the case of Bernoulli's Lemniscate scanning is due to the shape of the Lemniscate itself. Whenever the system manages to enter in the lock range, there are four transitions in this area, resulting in the increase of number of transitions.

As the parameter a increases, the number of the transitions increases due to the greater probability of the system to enter in lock range.

The trend of results, is similar for all given scenarios since the acquisitions error for ship motions together with errors of acquisition and orbital propagation for the satellite affect the results, unlike the ship motions.

\section{Actuators}

As indicated above, the scanning technique will be implemented by the optical system with actuators linked to the mirror reflecting the laser beam.

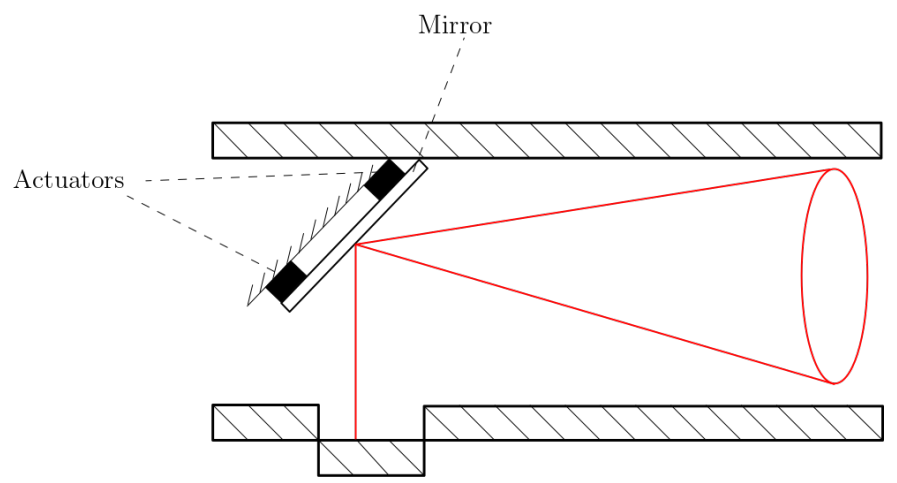

Figure 22: Optical System section.

The objective of this chapter is to make the choice of the actuator starting from the specifications of $r_{s}($ or $a)$ and $\omega$ that define the scanning technique.

First we need to introduce the features of the mirror:

\begin{tabular}{llll}
\hline Feature & Value & Description & Unit \\
\hline$r_{\mathrm{m}}$ & 0.015 & Mirror radius & {$[\mathrm{m}]$} \\
$\mathrm{s}$ & 0.01 & Mirror thickness & {$[\mathrm{m}]$} \\
$\mathrm{m}$ & 0.016 & Mirror mass & {$[\mathrm{kg}]$}
\end{tabular}

Table 7: Mirror features.

Piezoelectric actuators, for example, can be chosen by consulting the online catalog [23].

Considering the mirror and scanning technique parameters, we can evaluate the characteristics of the acuators such as Travel Range, Resonant Frequency and Pull Force.

Considering $r$ equal to $r_{s}$ in case of Conical scanning and $r$ equal to $a$ in case of Bernoulli's Lemniscate scanning, we can write the equation of Travel Range:

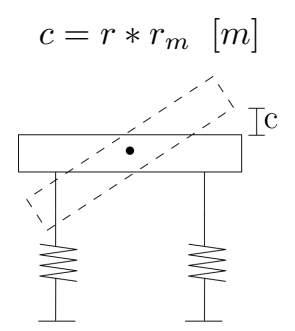

Figure 23: Travel Range.

Pull Force:

$$
I * \ddot{\alpha}=F * r_{m}
$$




\begin{tabular}{ll}
\hline Parameter & Description \\
\hline $\mathrm{I}$ & Mirror inertia $\left[\mathrm{Kg} \cdot \mathrm{m}^{2}\right]$ \\
$\ddot{\alpha}$ & Scanning acceleration $\left[\mathrm{rad} / \mathrm{s}^{2}\right]$ \\
$\mathrm{F}$ & Pull Force $[\mathrm{N}]$
\end{tabular}

Table 8: Equation parameters.

$\ddot{\alpha}$ is obtained deriving twice times the equation of motion that describes the Conical or Bernoulli's Lemniscate scanning:

- Conical Scanning:

$$
\alpha=r_{s} * \cos (w * t)[\mathrm{rad}]
$$

- Bernoulli's Lemniscate Scanning:

$$
\alpha=\frac{a * \cos (w t)}{1+\sin ^{2} w t}[\mathrm{rad}]
$$

In general, we can consider the maximum value of $\ddot{\alpha}$ :

$$
\ddot{\alpha}=\left|r * \omega^{2}\right|\left[\mathrm{rad} / \mathrm{s}^{2}\right]
$$

With $r$ equal to $r_{s}$ for the Conical scanning and $r$ equal to $a$ for the Bernoulli's Lemniscate scanning. Before considering the Inertia I, we have to consider how the rotation of the mirror is:

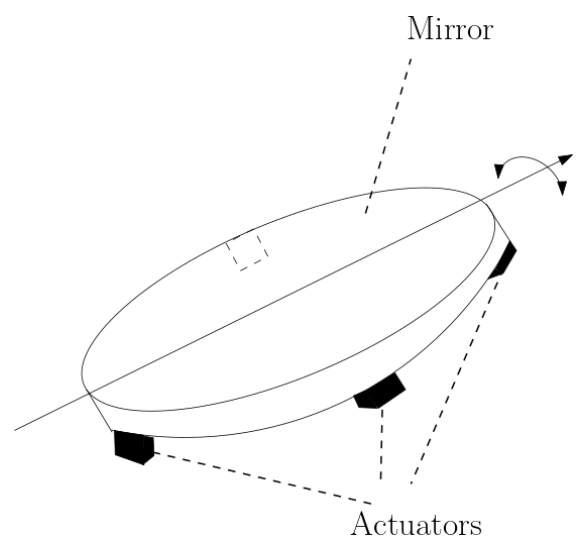

Figure 24: Mirror Rotation.

The mirror can be approximated as a small cylinder that rotates around the y axis:

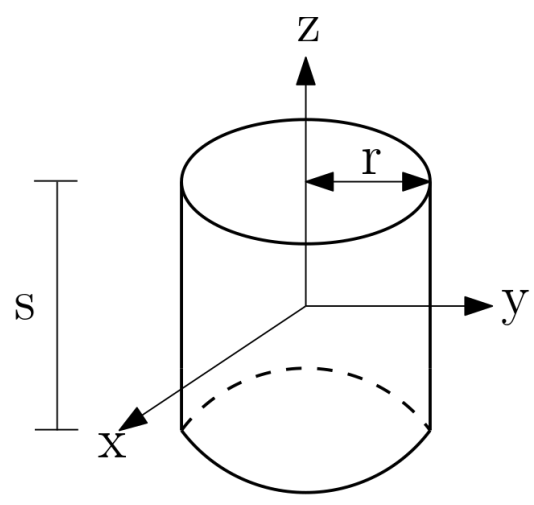

Figure 25: Cylinder axis of inertia.

$$
I_{y}=\frac{1}{12} *\left(3 * r_{m}^{2}+s^{2}\right)=1.15 * 10^{-6}\left[k g * m^{2}\right]
$$


Then, it is possible to define the Pull Force:

$$
F=\frac{I * r * \omega^{2}}{r_{m}}[N]
$$

Taking into account scanning results and actuators catalog some considerations can be made [23]:

- the Pull Force varies with the square of $\omega$;

- in order to have a high number of transitions in the lock range a high frequency will be necessary.

Consulting the online catalog of the manufacturer of piezoelectric actuators[24], the P-212 series can be considered:

\begin{tabular}{lllll}
\hline & P-212.1S & P-212.2S & P-212.4S & P-212.8S \\
\hline Travel range $[\mu \mathrm{m}]$ & 15 & 30 & 60 & 120 \\
Resonant frequency $[\mathrm{kHz}]$ & 17 & 12 & 7 & 4.5 \\
Pull Force $[\mathrm{N}]$ & 300 & 300 & 300 & 300
\end{tabular}

Table 9: Piezoelectric actuators series P-212 parameters.

Considering the equation 14 and the values of table 9, if the Travel Range value is low, then the value of $r$ is low. Consequently, if the value of $r$ is low, then the Conical Scanning cannot reach the lock zone, while Lemniscate scanning leads to a low number of transitions $(\simeq 20)$.

Considering $r=0.007[\mathrm{rad}]$ and $\omega=3500[\mathrm{~Hz}]$ :

\begin{tabular}{lll}
\hline Parameter & Value & Unit \\
\hline Travel Range & 108 & {$[\mu \mathrm{m}]$} \\
Pull Force & 125.7 & {$[\mathrm{~N}]$}
\end{tabular}

Table 10: Results for $\mathrm{r}=0.007[\mathrm{rad}]$ and $\omega=3500[\mathrm{~Hz}]$.

Considering these results and the frequency of $3500[\mathrm{~Hz}]$ the choice falls on the actuator P-212.8S.

\begin{tabular}{ll}
\hline Scanning type & Number of transitions \\
\hline Conical Scanning & $\simeq 400$ \\
Lemniscate Scanning & $\simeq 8000$
\end{tabular}

Table 11: Number of transitions for every scanning technique.

Further solutions can be achieved with different values of $\omega$ and $r$.

\section{Results discussion}

The intent to provide the scanning technique by rotating the mirror inside the optical system can be supported by the following calculations.

Supposing we want to provide the scanning technique with the whole PS Pointer:

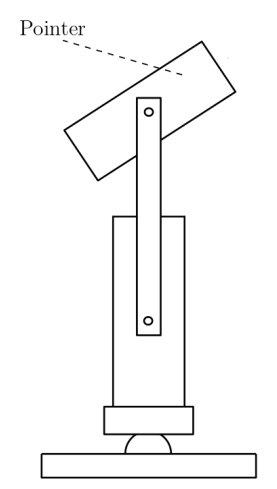

Figure 26: PS Model. 
We can define some Pointer features:

\begin{tabular}{llll}
\hline Feature & Value & Description & Unit \\
\hline$r_{\mathrm{p}}$ & 0.05 & Pointer radius & {$[\mathrm{m}]$} \\
$\mathrm{s}$ & 0.3 & Pointer heigth & {$[\mathrm{m}]$} \\
$\mathrm{m}$ & 2 & Pointer mass & {$[\mathrm{kg}]$}
\end{tabular}

Table 12: Pointer Features.

Considering the calculations of the previous chapter, we can calculate the force to set in motion the pointer at desired frequency:

\begin{tabular}{lll}
\hline Parameter & Value & Unit \\
\hline Force & 550 & {$[\mathrm{kN}]$}
\end{tabular}

Table 13: Results for $r=0.007$ [rad] and $\omega=3500[\mathrm{~Hz}]$.

The resulting Pull Force of 550 [kN] corresponds to 55 [ton] to be set in motion.

This would lead to the usage of large actuators that are not suitable for the requested application.

Considering the scanning technique provided by the mirror inside the optical system, we can draw some conclusions.

By evaluating the initial results and those referred to the scanning, the satellite's locking is feasible in case such scanning techniques are adopted.

The trend of results is similar for all given scenarios since the acquisitions error for ship motions together with errors of acquisition and orbital propagation for the satellite affect the results, unlike the ship motions.

The choice of the actuator, as described in the previous chapter, comes from the search for a good compromise in terms of Travel Range and adoptable frequency (frequency $\neq$ Resonant frequency).

For high values of frequency, there are low values of Travel Range. However, low values of $r$ lead to an undesirable number of transitions in the lock range which could compromise the satellite's locking.

Therefore, the results obtained in terms of frequencies and analyzed $r$ values can be considered adequate. 


\section{References}

[1] Rødseth, $\varnothing$. J.: From Concept to Reality: Unmanned Merchant Ship Research in Norway. IEEE Underwater Technology. (2017) 1-3.

[2] MUNIN. n.d. About MUNIN - Maritime Unmanned Navigation through Intelligence in Networks [Online]. Accessed 8 february 2022. http://www.unmanned-ship.org/munin/ about/

[3] Bedington, R.; Arrazola, J. M.; Ling, A.: Progress in satellite quantum key distribution. Nature Partner Journals. (2017) 2-4.

[4] Bennett, C. H.: Quantum cryptography: Public key distribution and coin tossing. International Conference on Computer System and Signal Processing, IEEE, 1984. (1984) 175-179.

[5] Tobias S-Manderbach, et al.: Experimetal Demostration of Free-Space Decoy-State Quantum Key Distribution over 144 km, Phys. Rev. Lett. 98 (2007) 010504.

[6] Toyoshima, M.; Takayama, Y.; Takahashi, T.; et al.: Ground-to-satellite laser communication experiments. IEEE Aerosp. Electron. Syst. Mag., 2008, 23, 10-18.

[7] Liao, S.K.; Cai,W.Q.; Liu,W.Y.; et al. : Satellite-to-ground quantum key distribution. Nature 2017, 549, $43-47$.

[8] Rabinovich,W.S.; Moore, C.I.; Mahon, R.; et al. : Free-space optical communications research and demonstrations at the US Naval Research Laboratory. Appl. Opt. 2015, 54, F189-F200.

[9] Wu, R.; Zhao, X.; Liu, Y.; Song, Y. : Initial pointing technology of line of sight and its experimental testing in dynamic laser communication system. IEEE Photonics J. 2019, 11, 1-8.

[10] He, D.; Wang, Q.; Liu, X.; et al. : Shipborne Acquisition, Tracking, and Pointing Experimental Verifications towards Satellite-to-Sea Laser Communication. Appl. Sci., 2019, 9, 3940,

[11] Doerry, Armin W., and Bickel, Douglas L.. Spotlight SAR Data Collection Geometry from ECEF Coordinates. United States: N. p., 2020.

[12] J. Sanz Subirana, J.M. Juan Zornoza and M. Hernández-Pajares, : Transformations between ECEF and ENU coordinates. Technical University of Catalonia, Spain. 2011 Available online: https://gssc.esa.int/navipedia/index.php/Transformations_between_ECEF_ and_ENU_coordinates

[13] Shuster, S. P.: A Survey and Performance Analysis of Orbit Propagators for LEO, GEO, and Highly Elliptical Orbits. Utah University All Graduate Theses and Dissertations. 2017. 6510. Available online: https://digitalcommons.usu.edu/etd/6510

[14] Dong, W.; Chang-yin, Z.: An Accuracy Analysis of the SGP4SDP4 Model. Chinese Astronomy and Astrophysics, 2010, 34, 69-76.

[15] J. Sanz Subirana, J.M. Juan Zornoza and M. Hernández-Pajares, : Ellipsoidal and Cartesian Coordinates Conversion. Technical University of Catalonia, Spain. 2011 Available online: https://gssc.esa.int/navipedia/index.php/Ellipsoidal_and_Cartesian_Coordinates_ Conversion

[16] Bulian, G., Moro, L., Brocco, E., Bresciani, F., Biot, M., Francescutto, A.: Using time domain nonlinear ship motion simulations to assess safety of people and cargo onboard a container vessel. In: Proc. 16th International Congress of the International Maritime Association of the Mediterranean (IMAM 2015) - Towards Green Marine Technology and Transport, 21-24 September 2015, Pula, Croatia, pp. 99-110 (Guedes Soares, Dejhalla \& Pavletić (Eds), CRC Press, Taylor \& Francis Group, London ISBN 978-1-138-02887-6), doi.org/10.1201/b18855-17

[17] Moro, L., Bulian, G., Brocco, E., Bresciani, F., Biot, M., Francescutto, A.: Failure analysis of container stacks by non-linear FE simulations under non-linear inertial loads. In: Proc. 16th International Congress of the International Maritime Association of the Mediterranean (IMAM 2015) - Towards Green Marine Technology and Transport, 21-24 September 2015, Pula, Croatia, pp. 745-754 (Guedes Soares, Dejhalla \& Pavletić (Eds), CRC Press, Taylor \& Francis Group, London ISBN 978-1-138-02887-6), doi.org/10.1201/b18855-100

[18] Bulian, G., Bresciani, F., Francescutto, A., Fucile, F.: Effect of large initial ship stability on ship safety: An example study. In: Proc. 26th European Safety and Reliability Conference (ESREL2016), 25-29 September 2016, Glasgow, Scotland, UK, in "Risk, Reliability and Safety: Innovating Theory and Practice", Walls, Revie \& Bedford (Eds), CRC Press/Balkema, Taylor \& Francis Group, London, 2017, pp. 2302-2309, ISBN 978-1-138-02997-2

[19] IACS, IACS Recommendation No.34 (Rev.1/Corr. 2001) - Standard Wave Data. International Association of Classification Societies (IACS), November 2001.

[20] BMT,: Global Wave Statistics. Primary contributors N. Hogben, N.M.C. Dacunha, G.F. Oliver. British Marine Technology, Unwin Brothers Limited, London, 1986.

[21] Bulian, G., Francescutto, A.: Experimental results and numerical simulations on strongly non-linear rolling of multihulls in moderate beam seas. Proc. Institution of Mech. Eng. - Part M - Journal of Engineering for the Maritime Environment, vol. 223, no. 3-4, 2009 , pp. 189-210.

[22] Rudaković, S., Bulian, G., Bačkalov, I.: Effective wave slope coefficient of river-sea ships. Ocean Engineering, Vol. 192, 2019, Article Number 106427, pp. 1-16, doi.org/10.1016/j.oceaneng.2019.106427

[23] https://www.physikinstrumente.com/en/products/ (visited on 08/20/2021).

[24] https://static.physikinstrumente.com/fileadmin/user_upload/physik_instrumente/files/datasheets/P-212-Datasheet.pdf 